\title{
Inverse activity of masticatory muscles with and without trismus: a brainstem syndrome
}

\author{
F. J E L A S I C A N D V. FR E I T A G \\ From the Department of Neurosurgery, and Department of Oral and Maxillofacial Surgery, \\ Medical Faculty, University of Saarland, Homburg-Saar, Germany
}

SUM M ARY Clinical and EMG findings in 10 cases of intrinsic brainstem lesions are reported with paradoxical activity of jaw closing muscles during jaw opening, with and without trismus. In five cases with trigeminal anaesthesia, the inverse activity of jaw closers is interpreted as a manifestation of disturbance in the central programming of mastication in the motor trigeminal area of the brainstem. Stretch reflex mechanisms and disinhibition of the trigeminal motor neurones play no part in the origin of inverse activity. The distinct brainstem syndrome can only be detected by EMG and the special clinical features.

Because of bilateral cortical innervation of the motor trigeminal nucleus, weakness of the masticatory muscles never occurs with unilateral cerebral lesions. In the case of bilateral supranuclear lesions there appears a paresis of masticatory muscles, the masseter reflex is hyperactive, and the stretch reflex mechanism occurs without trismus. In some cases of hemispheric and pontomesencephalic lesions unilateral inverse activity of jaw closing muscles with or without trismus was observed by Jelasic and Freitag (1974), a condition which was interpreted as a sign of disturbed programming of masticatory movements and of their co-ordination within the mesencephalic nucleus of the fifth nerve. We have now observed 10 cases of inverse, paradoxical activity of the jaw closing muscles, and present our further investigations.

\section{Methods}

The cases will be divided into two groups, one with and one without trismus.

\section{CASES WITH TRISMUS}

Seven cases with neurogenic trismus have been observed and three of these are presented.

Case 1 HL was a 42 year old man who had sustained a left frontotemporal fracture of the base

Address for correspondence and reprint requests: Professor Dr med. F. Jelasic, Neurochirurgische Universitatsklinik, D-6650 HomburgSaar, Bundesrepublik Deutschland.

Accepted 23 February 1978 of the skull with a traumatic carotid aneurysm in the region of the carotid syphon. There was left sided amaurosis, deafness, paralysis of the medial rectus and superior rectus, paralysis of the facial nerve, and complete anaesthesia in all three divisions of the left trigeminal area. The masseter did not contract either in jaw opening or in jaw closing, and trismus was present. On opening the jaw it deviated to the left. There was normal reflex activity in legs and arms, and no signs of spasticity or of involvement of upper motor neurones. Two months after the accident EMG showed no activity in the left masseter, with the mouth open or closed. Six months later there was inverse activity in the left masseter-that is, during jaw opening there was marked activity, but on closing the jaw only rare motor units were recorded. In the right masseter the EMG pattern was normal (Fig. 1). We could not elicit a masseter reflex, or reflex inhibition upon stimulation of perioral skin. One year later trismus disappeared.

Case $2 \mathrm{KL}$ was a 68 year old male with complete perceptive and expressive aphasia lasting one year due to cerebral sclerosis with cerebral vascular insufficiency. He had severe trismus which only disappeared when yawning. Reflex status was normal and symmetrical. There was no hemiparesis, but severe intellectual deficiency was present. During jaw closing the EMG showed marked activity in both masseters. At jaw opening there was intense activity in the right masseter, but the left masseter showed only two motor unit potentials (Fig. 2). Phasic masseter reflex was normal; 


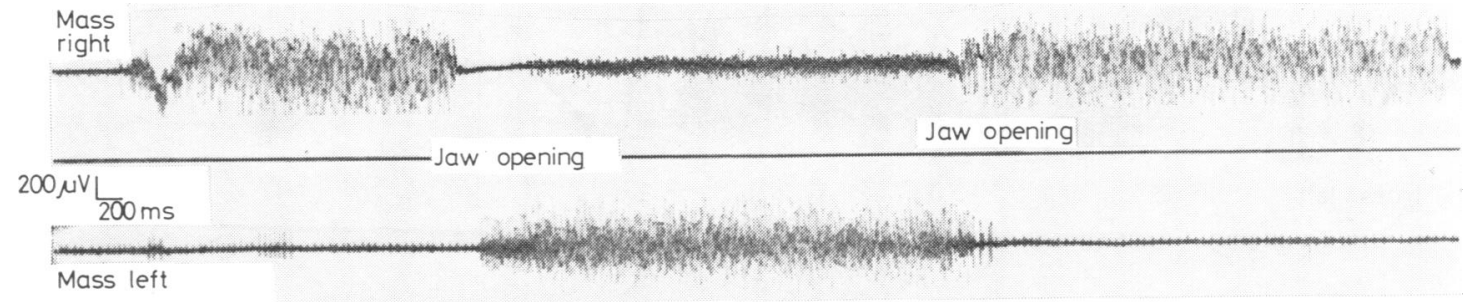

Fig. 1 Sensorimotor paresis of left trigeminal nerve. However, on this side (lower trace) at jaw opening there is intense activity, at jaw closing only rare motor units.

the silent period during jaw closing and opening was $125 \mathrm{~ms}$ (Fig. 2).

Case $3 \mathrm{BH}$, a 58 year old woman, had had a meningioma of the right pontocerebellar angle operated on. Severe trismus occurred on jaw opening with strong contraction of the right masseter. All divisions of right trigeminal territory showed severe hypaesthesia, but no anaesthesia. The phasic masseter reflex was absent and there was paralysis of the right sixth and seventh cranial nerves, paresis of the soft palate and pharyngeal muscles on the right side, and severe cerebellar ataxia, but no pyramidal tract signs. The EMG at rest and during jaw closing showed no activity in the right masseter and right medial pterygoid muscles as expected with sensorimotor paresis of the trigeminal nerve. But on jaw opening intense activity appeared in masseter and medial pterygoid muscles as though these muscles were jaw openers and not closers. Simultaneous registration from the right and left masseter recorded alternating activity of the right and left masseters (Fig. 3) as though these muscles were antagonists. Passive opening by pressure on the jaw did not evoke activity in the right masseter. Two years later trismus had diminished and activity on jaw closing had reappeared in the right masseter, but there was still some activity on jaw opening (Fig. 3).

\section{CASES WITH INVERSE ACTIVITY WITHOUT TRISMUS}

Three cases were observed and two are presented.

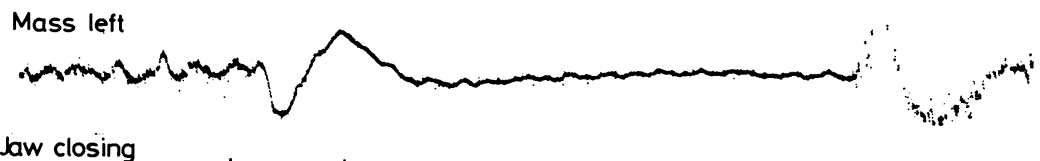

Mass right
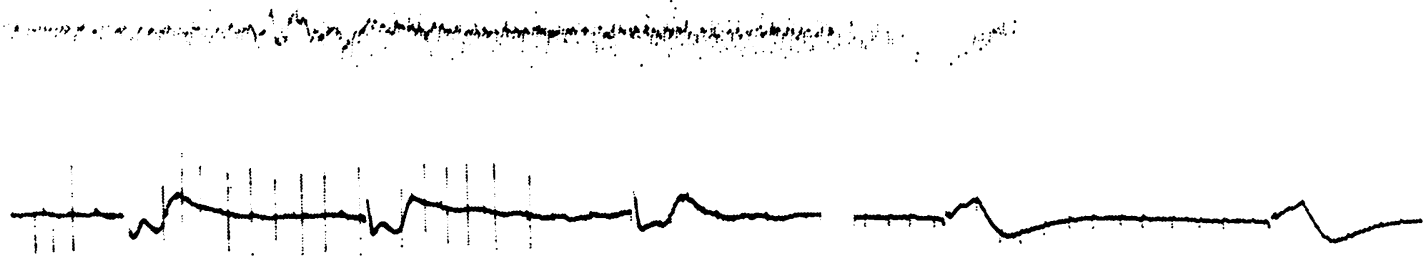

Mass left

Jaw opening

Mass right

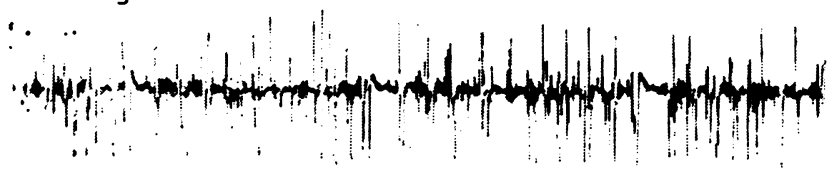

Jaw closing

Fig. 2 Top: at jaw opening marked activity in right masseter (side of trismus, lower trace). Bottom: masseter reflex and silent period on the right side (side of trismus, lower trace), normal =125 ms. 

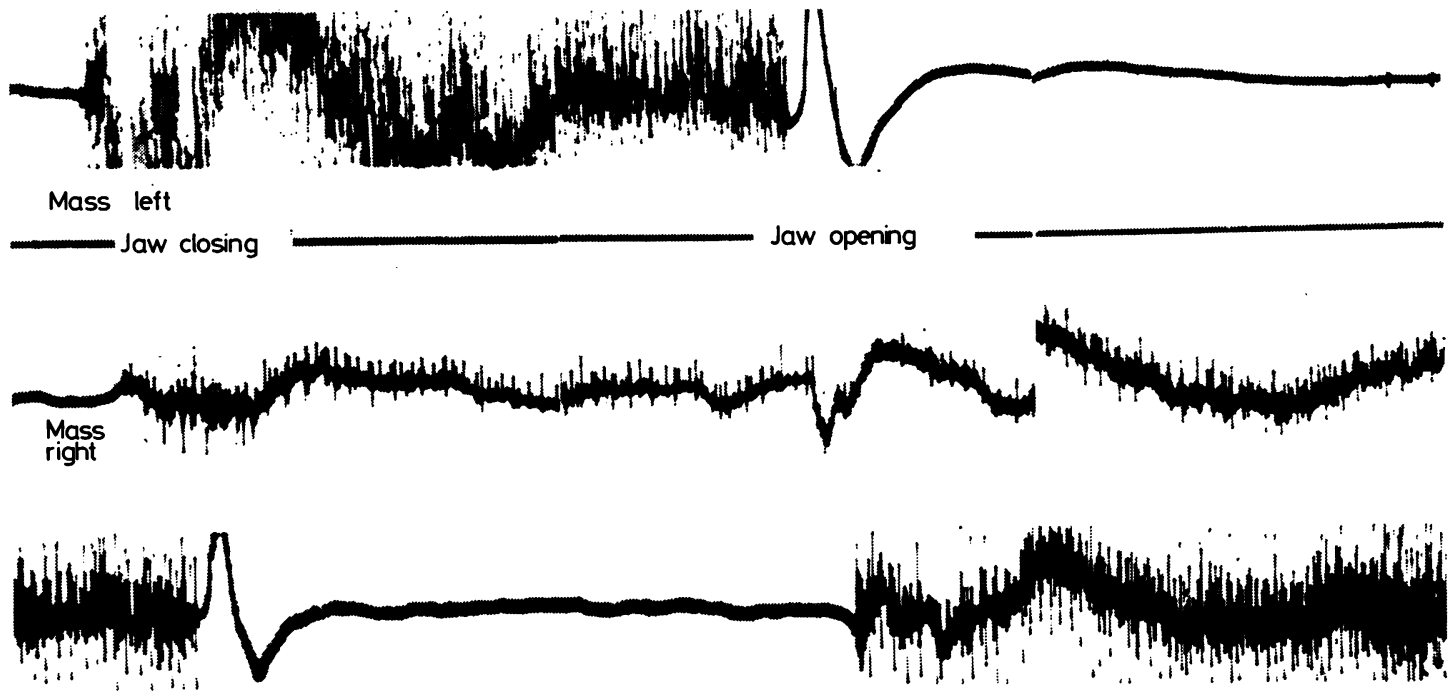

Mass left

Jaw closing maw opening

Jaw closing

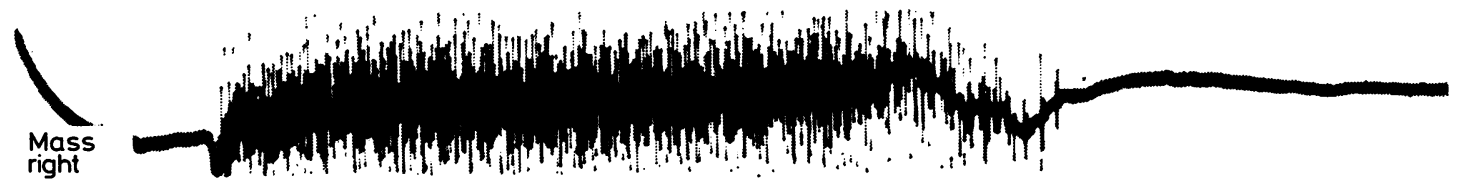

Fig. 3 Bottom: paresis of the right motor and sensory trigeminal nerve. At jaw closing no activity, at jaw opening intense paradoxical activity (lower trace). Top: two years later trismus diminished but some activity at jaw opening was still present.

Case 4 EB, a 23 year old woman, had an operation for chondroma of the clivus. Postoperatively and for many months she had complete paralysis of the left third, fourth, and sixth cranial nerves, and anaesthesia in all three divisions of the left trigeminal nerve territory. The left masseter did not contract on closing the mouth voluntarily. There was no trismus. Jaw closing produced marked EMG activity in the left and right masseter, weaker on the left. On jaw opening there was no EMG activity on the right side (not affected) but marked activity on the paretic left side (Fig. 4). The masseter reflex was normal on the right side with a silent period of $100 \mathrm{~ms}$. On the left side the reflex response was not registrable.

Case 5 GL, a 52 year old woman, had a pontine tumour causing paralysis of the left sixth nerve, nystagmus to the left side, cerebellar ataxia and paresis of the left vagus. On the left side there was an extensor plantar response, intention tremor, and hypacusis. No trismus was present. Voluntary activation of the masseter was normal, and the masseter reflex was moderately hyperactive. On jaw closing intense EMG activity was recorded in both masseters, on jaw opening there was intense activity in the left masseter (tumour side); in the right masseter activity ceased (Fig. 5).

Ten cases were observed, in which during jaw opening there was marked EMG activity in the jaw closing muscles. In seven cases trismus also appeared. In nine cases inverse activity was unilateral, in one bilateral. In four cases inverse activity was complete-that is, the jaw closing muscles were exclusively activated on jaw opening, but not on jaw closing. In six cases inverse activity was incomplete-that is, the jaw closers were activated normally on jaw closing but also inversely on jaw opening. Trigeminal sensation was absent or greatly diminished in five cases. Signs of an upper motor neurone lesion were observed in only three cases.

\section{Discussion}

Jaw closing muscles (masseter, temporalis, and 


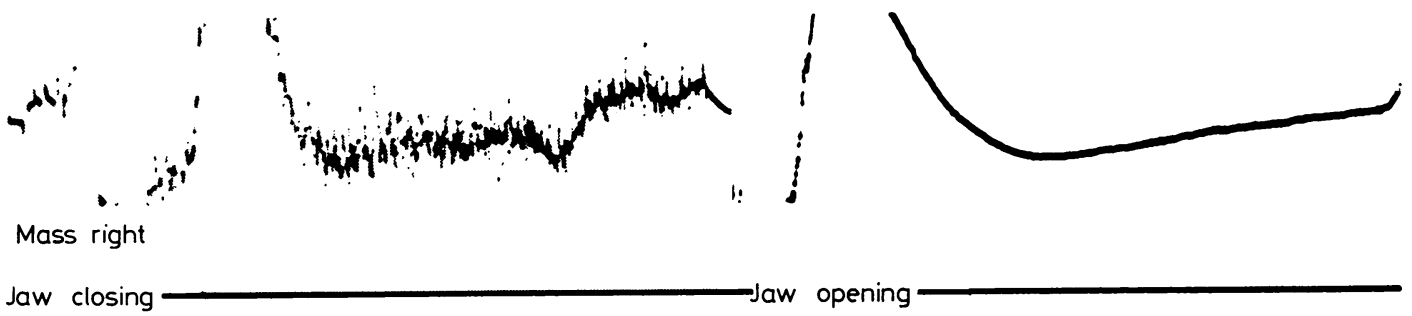

Mass left
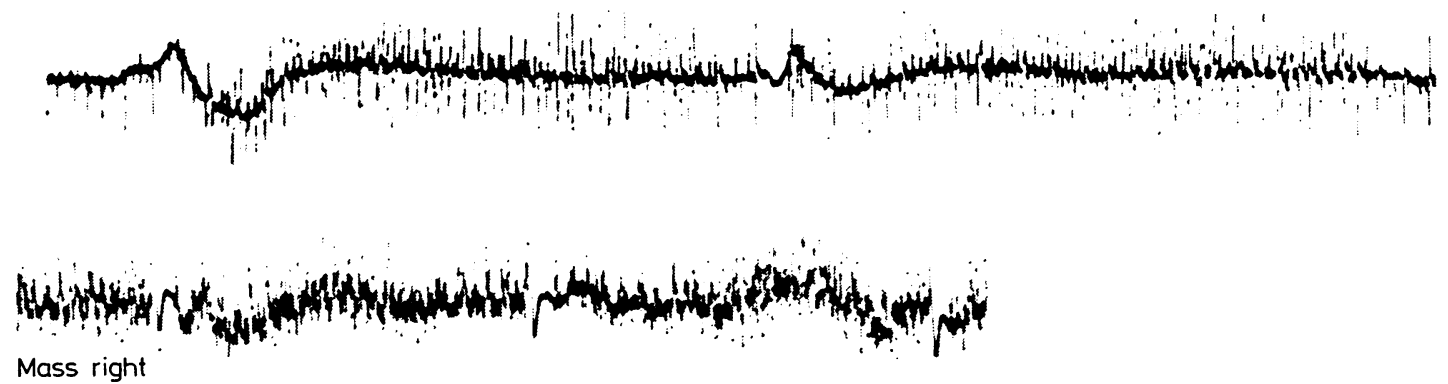

Jaw closing

Mass left

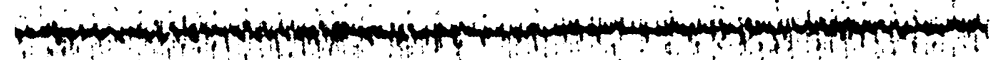

Fig. 4 Top: paresis of the left sensory and motor trigeminal nerve. On this side at jaw opening there is intense activity (lower trace), at jaw opening there iscomplete rest in the right masseter (upper trace). Bottom: on the side of inverse activity (left side, lower trace) masseter reflex does not register.

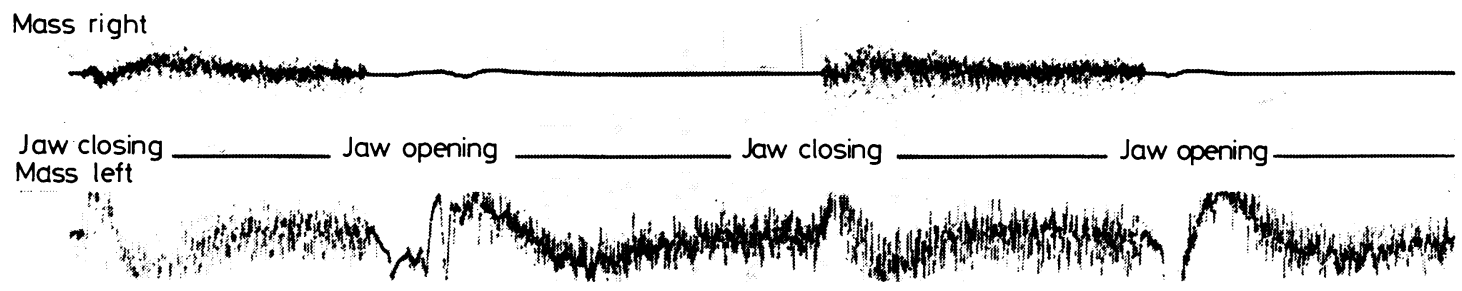

Fig. 5 In the left masseter marked activity at jaw closing and opening (lower trace), in the right masseter complete rest at jaw opening (upper trace).

medial pterygoid) and jaw opening muscles (mylohyoid, lateral pterygoid, and digastric) are antagonists. Activation of one group results in inhibition of the other (Carlsöö, 1952; Angelone et al., 1960; Møller, 1966). Only upon forced jaw opening can some activity of jaw closers be observed because of a protective function of these muscles against dislocation of the jaw. When a local irritative process in the jaw muscles, in the mouth, or in the temporomandibular joint, tetanus, or an intoxication with psychopharmaceuticals can be excluded, a centrally evoked trismus is postulated to be due to the lack of some inhibitory effect upon the motor cells in the nucleus, as with the stretch reflex mechanism in cases with upper motor neurone lesions or to lack of Renshaw inhibition of the lower motor neurone (Struppler et al., 1963). This similarity between spastic contracture in extremities and spastic contracture of jaw muscles or another hypothetical disinhibition 
of the motor cells could be accepted in trismus if spastic features or disinhibition of motoneurones could be proved. To investigate whether the inverse activity of jaw closers is due to disinhibition of the motor trigeminal nucleus based on an upper motor neurone lesion or to some other factors, we shall discuss the observed cases in two groups (a) without signs of upper motor neurone involvement and (b) with upper motor neurone involvement.

CASES OF INVERSE ACTIVITY WITHOUT SIGNS EITHER OF UPPER MOTOR NEURONE LESION OR OF MOTOR NEURONE EXCITATION

A lesion of the upper motor neurone can be accepted if there are (1) integrity of reflex arc-that is, alpha-motor neurones, afferent fibres, and muscle spindle inputs must be undamaged, (2) exaggerated muscle stretch reflexes and positive pathological reflexes, and (3) paresis of affected muscles.

In five out of seven cases without signs of upper motor neurone lesion there was anaesthesia of all three divisions of the trigeminal nerve on the side of inverse activity of jaw closers (in two cases due to a Dandy root section; in one case the Gasserian ganglion was destroyed by tumour; in one case there was a meningioma of the pontocerebellar angle; in one case a fracture of the skull with complete ophthalmoplegia). In all five cases with trigeminal anaesthesia there was no doubt about interruption of the reflex arc. A stretch reflex mechanism can, therefore, be excluded as a cause of trismus.

In all cases with trigeminal anaesthesia the masseter reflex and faciotrigeminal reflex were absent and could not be registered by EMG, as would be expected with sensory denervation of the muscles. On the other hand, it is well known that spasticity and exaggerated reflexes can be abolished by cutting posterior roots to interrupt afferent neurones. In our cases of trigeminal anaesthesia the motor neurones were unaffected. There was no atrophy, the contractibility and voluntary activation of the jaw closers and jaw openers were preserved, but it happened in a paradoxical, inverse way. There were no signs of irritation of the motor nucleus. All afferent inputs from peripheral sensory receptors and from spindles were interrupted. Hyperactivity of motoneurones due to the lack of Renshaw inhibition as in cases of tetanus cannot be accepted because in tetanus a strong activity of the closing muscles can be provoked by voluntary activation, or by any manipulation and perioral stimulation, but the excitatory response is a prolonged contraction of jaw closing muscles which cannot be interrupted voluntarily at any moment. The jaw closers react to any excitation with a strong and prolonged contraction followed by a strong closure of the jaw which can damage teeth and tongue. In our five cases with trigeminal anaesthesia such excitability did not exist, the jaw closing muscles did not close the jaw, their voluntary contraction appeared only on opening and not on closing the jaw. This means that there was an inverse but not intensified normal activity. Furthermore, there was no resting activity as is usual with lack of Renshaw inhibition.

The possibility that the spindle afferent fibres could activate the motor neurone cell antidromically can also be excluded because the antidromic impulses cannot compensate for the normal input from the spindles through afferent pathways. On the other hand, in nine of 10 cases the inverse activity was unilateral, while in the case of hypothetical antidromic activity a bilateral inverse activity should be expected to be much more intense on the unaffected side because on that side the alpha motor neurones can be activated through normal sensory fibres and accessory motor antidromic impulses. In Fig. 4 we can see that on the inverse activity (left masseter) with trigeminal anaesthesia the silent period could be evoked only on the unaffected side but not on the side where the spindle afferent pathways had been completely destroyed. This is in accordance with results of electrophysiological study in man (Bratzlavsky, 1973) which revealed that a silent period in jaw closers can be evoked only if the primary spindle afferent pathway is preserved.

With regard to paresis of the involved muscles as a factor in trismus, in every spastic condition a paresis of involved muscles is also postulated. A strong spasticity cannot exist without considerable paresis. In six cases the voluntary contraction of the jaw closers was as strong during jaw closing as during opening (Fig. 5, inverse activity without trismus). Furthermore, the activity could be initiated and interrupted at any time and jaw closing was not impaired. A paresis cannot be accepted under such circumstances. In two of our cases abolition of trismus was observed during yawning. This speaks against a stretch reflex mechanism, because if activation of the muscle spindles via a stretch reflex were the cause of trismus, an intensification, not cessation, of the trismus would be expected during yawning.

This observation shows that in the cases where afferent pathways were interrupted, the masseter reflex was abolished and where no evidence of paresis of jaw closers existed, spasticity or any other disinhibition cannot be the explanation for inverse activity of jaw closers during jaw opening. 
INVERSE ACTIVITY IN CASES OF UPPER MOTOR NEURONE LESIONS

In the first case (case 5, pontine tumour, Fig. 5) Babinski's sign was present on the side of inverse activity of jaw closers without trismus. In the second case (traumatic brainstem lesion) there was spasticity on both sides with a right sided hemiparesis, and trismus on the left side with intense activity of jaw closers during both opening and closing of the jaw on the left side. In the third case (stenosis of a vertebral artery) there was trismus with marked activity on closing and opening the jaw on the left side and hemiparesis on the right side. In all these three cases with inverse activity the voluntary contraction of jaw closers was normal. Therefore a spastic contraction in the absence of paresis cannot be accepted. In addition, the inverse activity was unilateral, while with an upper motor neurone lesion a bilateral increase of the stretch reflexes would be expected because of the bihemispheric innervation of the motor trigeminal nerve. Monohemispheric innervation of the trigeminus could be postulated as an anatomical anomaly, but in such a case paresis of trigeminal muscles should be expected.

\section{Conclusion}

The clinical, EMG, and theoretical analysis of the cases presented shows that intense activity of jaw closers during jaw opening with or without trismus is a special disturbance within the trigeminal motor nucleus which cannot be interpreted as a manifestation of stretch reflex mechanisms or of an excitation of the motor neurone. It is difficult to postulate the same nature of dysfunction in all 10 cases. But there is no doubt that in five cases with trigeminal anaesthesia the observed inverse activity cannot be a result either of proprioceptive sensory feedback from the periphery or of alternating reciprocal reflexes-that is, rhythmic activity of agonists and antagonists initiated by stretch. It has been postulated from experiments in animals (Lund, 1976; Taylor, 1976) that rhythmical alternating movements, such as jaw opening and closing during the chewing cycle, are controlled basically by a pattern generator within the brainstem. The activation of this generator would occur from a corticobulbar input and from proprioceptive feedback from the periphery, although returning information from peripheral sensory receptors is not obligatory as demonstrated by our cases where peripheral input was completely abolished. There is also a voluntary activity of these muscles, different from cyclical masticatory movement, which is cortically controlled and which can be interrupted or started at any moment. Because, in our cases, the rhythmic chewing movements were only quantitatively reduced but not abolished and voluntary activation of jaw closing muscles was inverse, our tentative conclusion is that the lesion is localised near that part of the motor trigeminal nucleus in the brainstem in which central programming of masticatory movements, independent of feedback from the periphery, is generated. The effect of this disturbance is inverse with paradoxical activity of jaw closers at the moment when these muscles should be inhibited during activity of jaw openers. The result is that jaw closers and jaw openers contract simultaneously instead of alternately. This is neither reflexive nor excitatory but an active innervatory appearance.

Paradoxical innervatory disturbances are not unknown in medicine. In the Duane-Stilling syndrome an activation of the lateral rectus muscle of the eyeball is elicited by contraction of the medial rectus, maximum contraction of the lateral rectus coincides with eye adduction, so eye adduction cannot be performed (similar to our cases where jaw closers are activated only through jaw opening), or the lateral rectus contracts normally and inversely, similar to our cases where jaw closers contract at jaw closing and jaw opening. In the Brown syndrome also there is a disturbance of synergy between the superior and inferior oblique muscles because of paradoxical innervation of the inferior oblique. The analogy between paradoxical activity of extraocular muscles and paradoxical activity of jaw muscles is obvious: abduction and adduction of the eye or jaw opening and closing are possible if the antagonists and agonists are alternately and not simultaneously activated. This alternating activation presupposes an intact central programming of mastication in the motor trigeminal area in the brainstem, which is not identical with rhythmic generation of the chewing cycle. Paradoxical activity of jaw closing muscles with or without trismus is a special, not very rare feature, which indicates a brainstem lesion.

\section{References}

Angelone, L., Clayton, J., and Brandhorst, W. (1960). An approach to quantitative electromyography of masseter muscle. Journal of Dental Research, 39, 17-23.

Bratzlavsky, M. (1973). Segmental reflex control of oral motor activities: an electrophysiological study in man. Acta Neurologica Belgica, 73, 145-153.

Carlsöö, S. (1952). Nervous co-ordination and the mechanical function of the mandibular elevators. Acta Odontologica Scandinavica, 10, Supplement 11. 
Jelasic, F., and Freitag, V. (1974). Trismus als symptom motorischer trigeminaler dyssenergie bei hirnstammprozessen. Journal of Neurology, 209, 287296.

Lund, J. (1976). Evidence for a central neural pattern generator regulating the chewing cycle. In Mastication. Edited by D. Anderson and B. Matthews. John Wright and Sons: Bristol.
Møller, E. (1966). The chewing apparatus. Acta Physiologica Scandinavica, 69, Supplement 280.

Struppler, A., Struppler, E., and Adams, R. (1963). Local tetanus in man. Archives of Neurology (Chicago), 8, 162-178.

Taylor, A. (1976). The role of jaw elevator muscle spindles. In Mastication. Edited by D. Anderson and B. Matthews. John Wright and Sons: Bristol. 